\title{
Cardiomiopatia de Estresse e Doença Coronária: a Coexistência das Duas Entidades Clínicas Pode Ser Possível
}

\author{
Hélder José Magalhães Ribeiro', Catarina Ferreira' ${ }^{1}$ Ana Baptista ${ }^{1}$, Pedro Magalhães', \\ Alberto Ferreira ${ }^{1}$, Ilídio Moreira ${ }^{1}$
}

\section{RESUMO}

A cardiomiopatia induzida por estresse é uma síndrome que mimetiza o infarto agudo do miocárdio. A presença de doença arterial coronária significativa é considerada um critério de exclusão de cardiomiopatia induzida por estresse; contudo, isso pode não ocorrer em todos os casos, dado que ambas as entidades podem coexistir. Apresentamos o relato de um caso de cardiomiopatia induzida por estresse em paciente com doença arterial coronária conhecida.

DESCRITORES: Cardiomiopatias. Estresse psicológico. Cardiomiopatia de takotsubo. Disfunção ventricular esquerda.

A cardiomiopatia induzida por estresse é uma síndrome caracterizada por dor torácica anginosa, habitualmente iniciada no contexto de estresse emocional ou físico intenso, associada a alterações eletrocardiográficas e enzimáticas que mimetizam o infarto agudo do miocárdio, e disfunção transitória do segmento apical do ventrículo esquerdo ou, menos frequentemente, do segmento médio. A presença de doença coronária significativa é considerada um critério de exclusão de cardiomiopatia induzida por estresse, de acordo com as recomendações atuais; contudo, estudos recentes apontam para uma coexistência possível das duas entidades.

\section{RELATO DO CASO}

Apresentamos o caso de uma mulher de 71 anos de idade, com múltiplos fatores de risco para ateros-

\footnotetext{
1 Centro Hospitalar de Trás-os-Montes e Alto Douro - Hospital de Vila Real - Vila Real, Portugal.

Correspondência: Hélder José Magalhães Ribeiro. Rua Actor Ruy de Carvalho, 8 - Vila Real, Portugal - 5000-542

E-mail: hjmribeiro@gmail.com

Recebido em: 26/7/2011 • Aceito em: 13/9/2011
}

\begin{abstract}
Stress Cardiomyopathy and Coronary Disease:

Possible Coexistance of Two Clinical Entities

Stress-induced cardiomyopathy mimics acute myocardial infarction. Significant coronary artery disease is generally considered an exclusion criterion for the diagnosis of stressinduced cardiomyopathy. However, this may not be the case for all patients, since both entities may coexist. We present a case of stress-induced cardiomyopathy in a patient with known coronary artery disease.
\end{abstract}

KEY-WORDS: Cardiomyopathies. Stress, psychological. Takotsubo cardiomyopathy. Ventricular dysfunction, left.

clerose, incluindo hipertensão arterial de longa data, diabetes melito tipo 2 há 10 anos, em uso de antidiabéticos orais, dislipidemia e obesidade grau 2, com índice de massa corporal (IMC) de $38 \mathrm{~kg} / \mathrm{m}^{2}$. Apresentava antecedentes de cardiopatia isquêmica com internação em agosto de 2010 por angina instável, cuja investigação revelou doença coronária significativa nos três vasos e função ventricular esquerda conservada (Figura 1). A cirurgia de revascularização do miocárdio foi proposta e aceita, e a paciente ficou aguardando, em seu domicílio, ser chamada para a cirurgia, permanecendo com angina de esforço classe II da Canadian Cardiovascular Society (CCS).

No final de fevereiro de 2011, durante a noite, e após estresse emocional intenso (assistiu à detenção policial de um de seus filhos, em sua própria casa), iniciou episódio de dor torácica anginosa intensa em repouso, parcialmente aliviada com nitratos sublinguais. Em decorrência da persistência da dor, recorreu ao serviço de urgência, sendo admitida ainda sintomática. O eletrocardiograma evidenciou ondas $Q$ em DIII e aVF, infradesnivelamento do segmento ST, e inversão da onda T de V2-V6 e em D1 e aVL (Figura 2). O estudo analítico inicial revelou elevação dos marcadores de necrose do miocárdio, sendo a paciente 

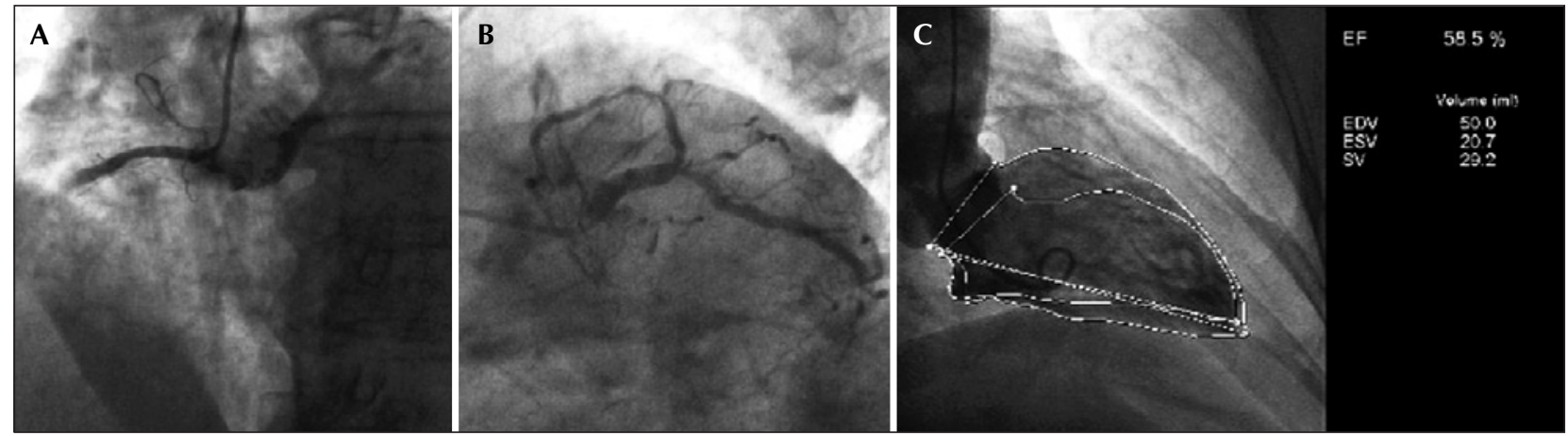

Figura 1 - Cateterismo cardíaco realizado em agosto de 2010, evidenciando doença coronária grave de 3 vasos e função ventricular esquerda globalmente conservada. Em A, coronária direita com oclusão na transição do segmento proximal para o segmento médio. Em B, coronária esquerda com estenose suboclusiva no segmento médio da descendente anterior e estenose significativa ostial da circunflexa. Em C, ventriculografia demonstrando função ventricular esquerda globalmente conservada, sem alterações da contratilidade segmentar.

internada na Unidade Coronária com diagnóstico de infarto agudo do miocárdio sem elevação do segmento ST. Evoluiu favoravelmente com resolução da dor (após início de nitratos endovenosos), estabilidade elétrica e pico de troponina $\mathrm{T}$ de 1,91 $\mathrm{ng} / \mathrm{ml}$. Contudo, apresentou tendência a hipotensão, sendo realizado ecocardiograma, que demonstrou discinesia apical com acinesia de todos os segmentos distais adjacentes, hipocinesia dos segmentos médios, hipercontratilidade dos segmentos basais e depressão moderada a grave da função sistólica do ventrículo esquerdo, com fração de ejeção média estimada em 32\% (Figura 3).

Realizado novo cateterismo cardíaco, que revelou discinesia apical e hipercontratilidade dos segmentos basais, que conferia ao ventrículo esquerdo o aspecto de balonamento apical. A doença coronária mostrou o mesmo padrão de acometimento dos 3 vasos, com as mesmas lesões encontradas no cateterismo realizado em agosto de 2010, com oclusão da coronária direita na transição do segmento proximal para o segmento médio, e leito distal parcialmente visualizado por circulação colateral homolateral, estenose significativa do segmento médio da descendente anterior e óstio da circunflexa (Figura 4). A paciente evoluiu clinicamente bem e no quinto dia de internação foi realizado ecocardiograma de controle, que demonstrou a normalização da função ventricular esquerda (Figura 5). O diagnóstico inicial foi corrigido para cardiomiopatia induzida por estresse.

\section{DISCUSSÃO}

A cardiomiopatia induzida por estresse, também conhecida por cardiomiopatia de takotsubo, foi descrita inicialmente por Satoh et al. ${ }^{1}$, no Japão. O nome deriva da forma de balão do ventrículo esquerdo, similar à armadilha de polvo utilizada no Japão (takotsubo, em japonês). A anormalidade mais comum é a hipocinesia segmentar apical do ventrículo esquerdo transitória associada a hipercinesia compensatória na pa-

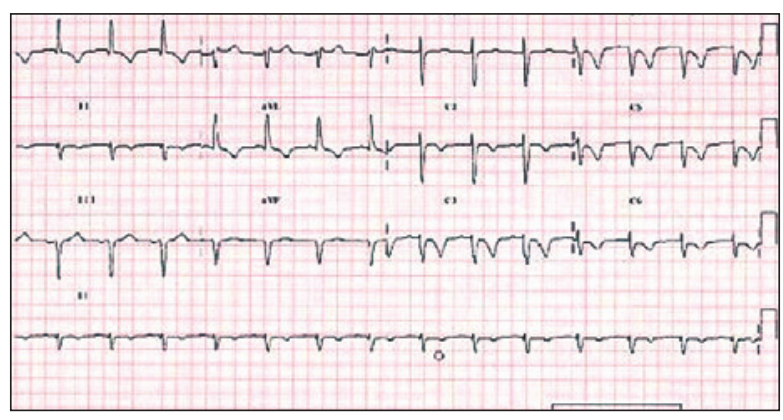

Figura 2 - Eletrocardiograma à admissão, evidenciando ritmo sinusal, ondas Q em DIII e aVF, infradesnivelamento do segmento ST, inversão da onda $T$ de $\mathrm{V} 2-\mathrm{V} 6$ e em D1 e aVL.

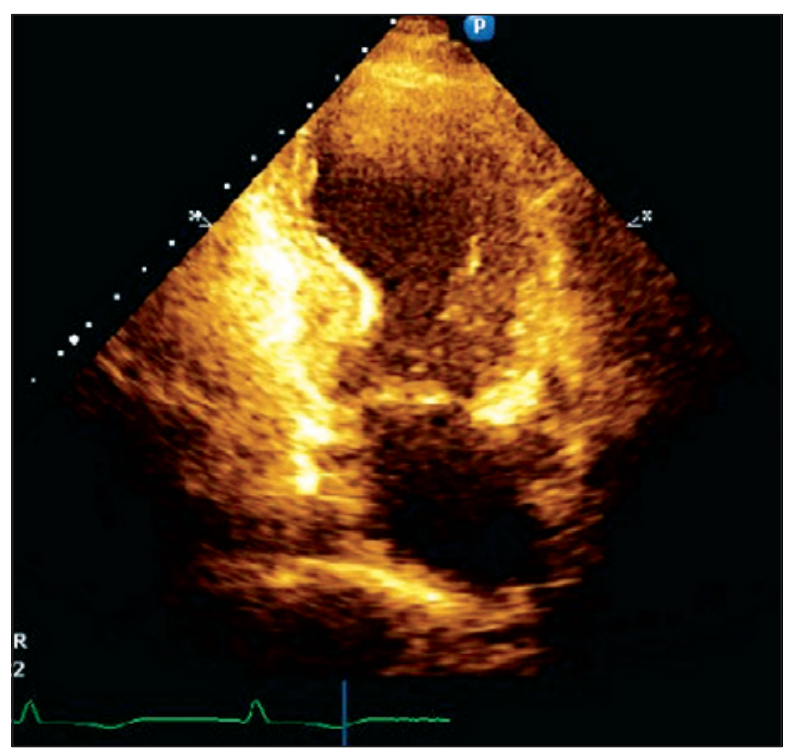

Figura 3 - Ecocardiograma à admissão, evidenciando discinesia apical e hipercontratilidade dos segmentos basais.

rede basal, produzindo a forma típica de balão durante a sístole. Menos frequentemente ocorre hipocinesia 

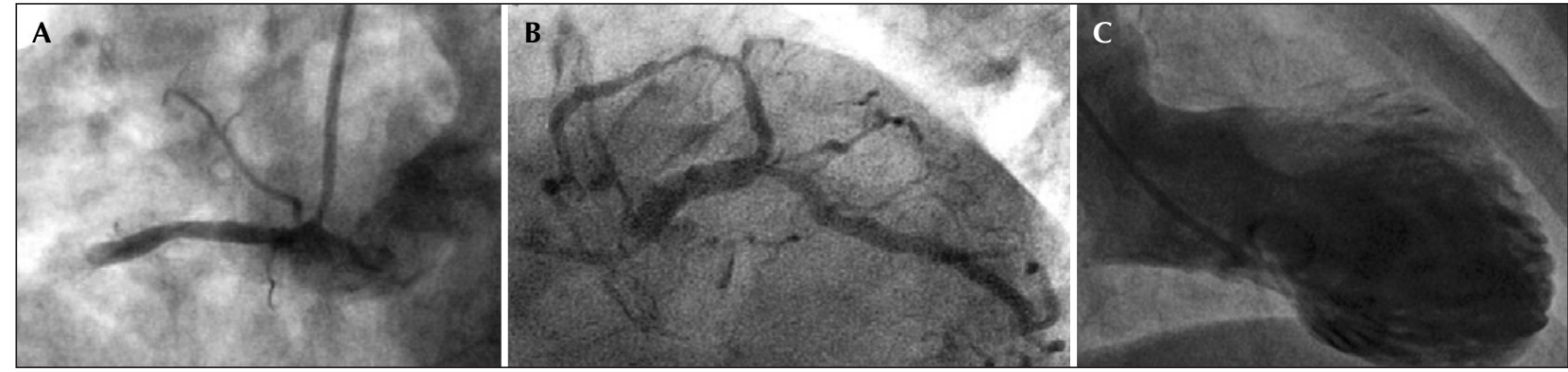

Figura 4 - Cateterismo cardíaco realizado em fevereiro de 2011, revelando doença coronária grave em 3 vasos (sobreponível ao cateterismo de agosto de 2010) e depressão grave da função ventricular esquerda com balonamento apical. Em A, coronária direita com oclusão na transição do segmento proximal para o segmento médio. Em B, coronária esquerda com estenose suboclusiva no segmento médio da descendente anterior e estenose significativa ostial da circunflexa. Em C, ventriculografia demonstrando depressão grave da função ventricular esquerda, com discinesia apical e hipercontratilidade dos segmentos basais.

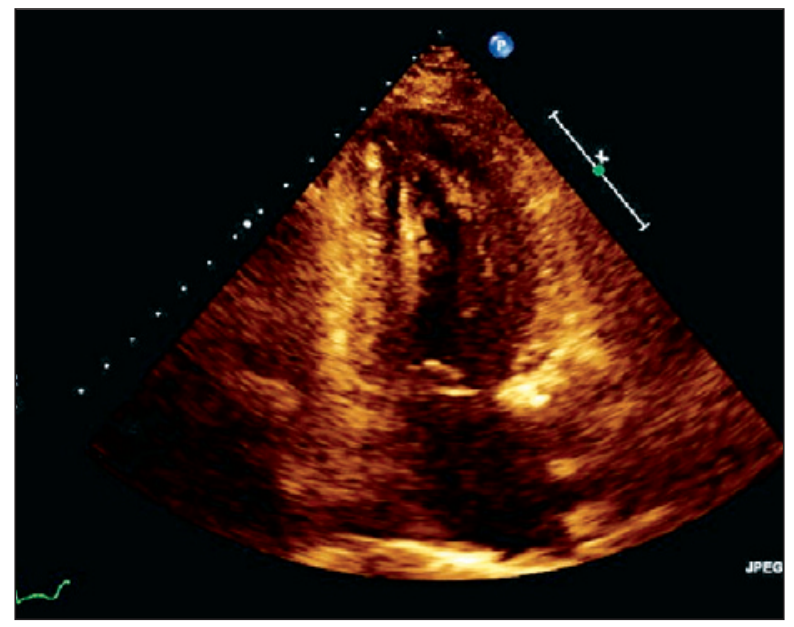

Figura 5 - Ecocardiograma realizado no quinto dia de internação, evidenciando ausência de alterações na contratilidade segmentar.

transitória mais evidente do segmento médio do ventrículo esquerdo (cerca de $40 \%$ dos casos), configurando uma forma atípica de cardiomiopatia induzida por estresse.

Essa entidade foi descoberta recentemente e como tal não existem muitos dados sobre sua prevalência. A partir de 2000, vários relatos e séries de casos têm descrito essa condição. ${ }^{2-4}$ A prevalência estimada de $1 \%$ a $2 \%$ é proveniente de pequenas séries de casos de pacientes com suspeita de síndrome coronária aguda, que foram incluídos numa grande revisão sistemática relatada por Gianni et al. ${ }^{5}$, e de um registo de 3.265 pacientes com síndrome coronária aguda e biomarcadores cardíacos elevados, relatado por Kurowski et al. ${ }^{6}$. É mais prevalente em mulheres que em homens (aproximadamente 90\%), exatamente o oposto do que ocorre na prevalência de doença arterial coronáriaa ${ }^{2,7,8}$, desconhecendo-se o motivo pelo qual essa condição afeta predominantemente as mulheres. Há relatos de que mulheres na pós-menopausa, entre 62 e 75 anos de idade, apresentam maior risco de desenvolver essa cardiomiopatia, sendo esse risco $<3 \%$ em mulheres com menos de 50 anos de idade. ${ }^{7,8}$

Existem incertezas quanto à fisiopatologia da cardiomiopatia induzida por estresse, porém acredita-se que possa ocorrer disfunção ou espasmo microvascular difuso induzidos pela libertação excessiva de catecolaminas desencadeada por estresse físico ou emocional ou por toxicidade direta das catecolaminas no músculo cardíaco, resultando em atordoamento miocárdico. ${ }^{5}$ Frequentemente são identificados fatores de estresse físico ou emocional desencadeantes, como morte inesperada de familiares ou amigos, abuso doméstico, catástrofe natural, perda de dinheiro ou falência, falar em público, e acidentes aéreos. ${ }^{2,8}$ No caso relatado, a paciente iniciou o quadro de dor torácica após assistir à detenção policial de um de seus filhos.

Não existe consenso quanto aos critérios diagnósticos da cardiomiopatia induzida por estresse. Os sintomas e o eletrocardiograma não têm valor preditivo suficiente para distinguir um paciente com cardiomiopatia induzida por estresse de um com infarto agudo do miocárdio, sendo a angiografia coronária o melhor método de diagnóstico para confirmar essa condição. ${ }^{8}$ Dessa forma, em 2004, investigadores da Clínica Mayo desenvolveram critérios diagnósticos, recentemente modificados, que têm sido comumente utilizados. Os quatro critérios apresentados a seguir devem ser preenchidos: 1) anomalia transitória da contratilidade segmentar, que se estende além da distribuição de um vaso epicárdico, habitualmente hipocinesia ou acinesia transitória do segmento médio do ventrículo esquerdo, com ou sem envolvimento apical (em geral com redução da fração de ejeção para valores entre 20\% e 49\%) e não raro associada a um fator estressante; 2) ausência de doença arterial coronária angiográfica significativa ou evidência angiográfica de ruptura de placa; 3) alteração eletrocardiográfica de novo (elevação do segmento ST, que é a mais frequente, localizada na parede anterior em cerca de $80 \%$ dos casos, e/ou inversão da onda T) ou elevação moderada da troponina 
cardíaca (que frequentemente não se correlaciona com a gravidade do comprometimento hemodinâmico); e 4) ausência de feocromocitoma ou miocardite..$^{2,5,8} \mathrm{~A}$ paciente preenchia todos os critérios descritos, com exceção da ausência de doença coronária. A presença de doença coronária significativa é geralmente considerada um critério de exclusão de cardiomiopatia induzida por estresse.

Estudo de Winchester et al. ${ }^{9}$, entretanto, sugere que $29 \%$ dos pacientes com cardiomiopatia induzida por estresse apresentam estenose superior a 50\% em pelo menos um vaso epicárdico. Kurisu et al. ${ }^{10}$, em outro estudo, demonstraram a presença de doença coronária significativa (estenose $>75 \%$ ) em pelo menos um vaso epicárdico em 10\% dos casos de uma série de 97 pacientes japoneses com diagnóstico de cardiomiopatia induzida por estresse. Mais recentemente, outro estudo, realizado por To et al. ${ }^{11}$, descreveu a prevalência de $15 \%$ de doença coronária moderada ou grave em uma série de 56 doentes com cardiomiopatia induzida por estresse. Assim, o caso apresentado corrobora a noção de que a cardiomiopatia induzida por estresse e a doença coronária não constituem entidades mutuamente exclusivas, podendo coexistir numa pequena proporção de doentes, e de que a exclusão de cardiomiopatia induzida por estresse, tendo por base apenas o achado incidental de doença coronária, não se justifica em todas as situações. Impõe-se uma decisão caso a caso, particularmente na presença de doença no segmento proximal da descendente anterior, em que cuidadosa avaliação deve ser realizada para analisar se o balonamento apical é causado pela doença coronária, com o objetivo de evitar revascularização coronária desnecessária. Este caso coloca em debate a necessidade de revisão dos critérios utilizados atualmente no diagnóstico dessa entidade, que, ao excluírem os pacientes com doença coronária, estão muito provavelmente subestimando a verdadeira incidência dessa doença.

\section{CONFLITO DE INTERESSES}

Os autores declaram não haver conflito de interesses relacionado a este manuscrito.

\section{REFERÊNCIAS}

1. Satoh H, Tateishi H, Uchida T. Takotsubo-type cardiomyopathy due to multivessel spasm. In: Kodama K, Haze K, Hon M, editors. Tokyo: Kagakuhyouronsya; 1990. p. 56-64.

2. Tsuchihashi K, Ueshima K, Uchida T, Oh-mura N, Kimura K, Owa M, et al. Transient left ventricular apical ballooning without coronary artery stenosis: a novel heart syndrome mimicking acute myocardial infarction. Angina Pectoris- Myocardial Infarction Investigations in Japan. J Am Coll Cardiol. 2001;38(1):11-8.

3. Kurisu $S$, Sato $H$, Kawagoe $T$, Ishihara $M$, Shimatani $Y$, Nishioka K, et al. Tako-tsubo-like left ventricular dysfunction with ST-segment elevation: a novel cardiac syndrome mimicking acute myocardial infarction. Am Heart J. 2002;143(3): 448-55.

4. Sato M, Fujita S, Saito A, Ikeda Y, Kitazawa H, Takahashi $M$, et al. Increased incidence of transient left ventricular apical ballooning (so-called "Takotsubo" cardiomyopathy) after the mid-Niigata Prefecture earthquake. Circ J. 2006; 70(8):947-53.

5. Gianni M, Dentali F, Grandi AM, Sumner G, Hiralal R, Lonn E. Apical ballooning syndrome or takotsubo cardiomyopathy: a systematic review. Eur Heart J. 2006;27(13):1523-9.

6. Kurowski V, Kaiser A, Von Hof K, Killermann DP, Mayer B, Hartmann F, et al. Apical and midventricular transient left ventricular dysfunction syndrome (tako-tsubo cardiomyopathy): frequency, mechanisms, and prognosis. Chest. 2007; 132(3):809-16.

7. Bybee KA, Kara T, Prasad A, Lerman A, Barsness GW, Wright RS, et al. Systematic review: transient left ventricular apical ballooning: a syndrome that mimics ST-segment elevation myocardial infarction. Ann Intern Med. 2004;141(11):858-65.

8. Prasad A, Lerman A, Rihal CS. Apical ballooning syndrome (Tako-Tsubo or stress cardiomyopathy): a mimic of acute myocardial infarction. Am Heart J. 2008;155(3):408-17.

9. Winchester DE, Ragosta M, Taylor AM. Concurrence of angiographic coronary artery disease in patients with apical ballooning syndrome (tako-tsubo cardiomyopathy). Catheter Cardiovasc Interv. 2008;72(5):612-6.

10. Kurisu S, Inoue I, Kawagoe T, Ishihara M, Shimatani $Y$, Nakama $Y$, et al. Prevalence of incidental coronary artery disease in tako-tsubo cardiomyopathy. Coron Art Dis. 2009; 20(3):214-8.

11. To AC, Kay P, Khan AA, Kerr AJ. Coronary artery anatomy and apical sparing in apical ballooning syndrome: implications for diagnosis and aetiology. Heart Lung Circ. 2010;19(4):219-24. 